OPEN

SUBJECT AREAS:

LASER MATERIAL

PROCESSING

COMPUTATIONAL METHODS

INTEGRATED OPTICS

ULTRAFAST LASERS

Received

16 September 2014

Accepted

28 November 2014

Published

7 January 2015

Correspondence and requests for materials should be addressed to J.S. (j.solis@io.cfmac. csic.es)

\title{
Rapid assessment of nonlinear optical propagation effects in dielectrics
}

\author{
J. del Hoyo' , A. Ruiz de la Cruz², E. Grace ${ }^{3}$, A. Ferrer ${ }^{4}$, J. Siegel' , A. Pasquazi ${ }^{5}$, G. Assanto ${ }^{6}$ \& J. Solis ${ }^{1}$
}

'Laser Processing Group, Instituto de Optica (CSIC), Serrano 121, Madrid 28006, Spain, ${ }^{2}$ Formerly at Laser Processing Group, Instituto de Optica (CSIC), Serrano 121, Madrid 28006, Spain, ${ }^{3}$ Formerly at Photonics Group, Blackett Laboratory, Imperial College London, Prince Consort Road, London SW7 2BW, UK, ${ }^{4}$ Institute of Quantum Electronics, ETH Zurich, 8093 Zurich, Switzerland, ${ }^{5}$ Department of Physics and Astronomy, University of Sussex, Sussex House, Falmer Brighton, BN1 9QH, UK, "NooEL-Nonlinear Optics and OptoElectronics Lab, CNISM and Department of Electronic Engineering - University "Roma Tre", Via della Vasca Navale 84, 00146 Rome, Italy.

Ultrafast laser processing applications need fast approaches to assess the nonlinear propagation of the laser beam in order to predict the optimal range of processing parameters in a wide variety of cases. We develop here a method based on the simple monitoring of the nonlinear beam shaping against numerical prediction. The numerical code solves the nonlinear Schrödinger equation with nonlinear absorption under simplified conditions by employing a state-of-the art computationally efficient approach. By comparing with experimental results we can rapidly estimate the nonlinear refractive index and nonlinear absorption coefficients of the material. The validity of this approach has been tested in a variety of experiments where nonlinearities play a key role, like spatial soliton shaping or fs-laser waveguide writing. The approach provides excellent results for propagated power densities for which free carrier generation effects can be neglected. Above such a threshold, the peculiarities of the nonlinear propagation of elliptical beams enable acquiring an instantaneous picture of the deposition of energy inside the material realistic enough to estimate the effective nonlinear refractive index and nonlinear absorption coefficients that can be used for predicting the spatial distribution of energy deposition inside the material and controlling the beam in the writing process.

$\mathrm{T}$ he propagation of high intensity laser beams (cw or pulsed) in dielectrics has been the subject of intense investigation since the early research on self-focusing ${ }^{1-3}$ and laser-induced breakdown in transparent materials ${ }^{4-6}$. Even at relatively low powers the combined effects of diffraction, nonlinear refraction and nonlinear absorption leads to a large variety of spatial and temporal effects ${ }^{7}$ ranging from self-focusing and self phase modulation to the formation of solitons ${ }^{8}$. The situation becomes more complex when the amount of energy absorbed during beam propagation gives rise to the formation of sufficiently dense electron plasmas to induce beam attenuation and self-defocusing ${ }^{5,9,10}$. Depending on the optical power, diffraction, space-time focusing/defocusing, group velocity and higher order dispersion phenomena, modulation instabilities, self steepening, Raman scattering, nonlinear absorption, plasma absorption and plasma defocusing can take place during propagation. A detailed description of these and other effects, like beam reshaping ${ }^{11}$ or propagation in presence of second order nonlinearities ${ }^{12}$ is outside the scope of this manuscript and can be found, for instance, in Refs. 7, 13.

In general, the description of the propagation of intense laser pulses in optical media is given by the Maxwell and constitutive equations with additional correction terms associated to nonlinear polarization and free carrier generation ${ }^{14,15}$. Under the assumption that the beam remains linearly polarized, in the slowly varying envelope (SVE) approximation with paraxial propagation conditions, the evolution of the field takes the form of the scalar nonlinear Schrodinger equation (NLSE $)^{14}$ :

$$
\begin{aligned}
& \frac{\partial A}{\partial z}=\frac{i}{2 k}\left(\frac{\partial^{2}}{\partial^{2} x^{2}}+\frac{\partial^{2}}{\partial^{2} y^{2}}\right) A+\frac{i k n_{2}|A|^{2}}{2 \eta_{0}} A-i\left(\left.\frac{\partial^{2} k}{\partial \omega^{2}}\right|_{\omega_{0}}\right) \frac{\partial^{2} A}{\partial^{2} t^{2}}-\frac{1}{2} \frac{W_{P I}(|A|) U_{i}}{|A|^{2}} A-\frac{\sigma}{2}\left(1+i \omega_{0} \tau_{c}\right) \rho A \\
& \text { (Term 1) } \\
& \text { (Term 2) } \\
& \text { (Term 3) } \\
& \text { (Term 4) } \\
& \text { (Term 5) }
\end{aligned}
$$


The propagation direction is taken along the $Z$ axis and the electric field is expressed as $\vec{E}=\frac{1}{2} A(x, y, z, t) \exp \left[i\left(k z-\omega_{0} t\right)\right] \vec{e}_{x}+$ $c c$. The pointwise intensity $\left.\left(<\mathrm{Wm}^{-2}\right\rangle\right)$ is given by $I=$ $\frac{c \varepsilon_{0} n}{2}|A|^{2}=\frac{n}{2 \eta_{0}}|A|^{2}$ where $\eta_{0}=\left(\varepsilon_{0} c\right)^{-1}$ is the vacuum impedance. The central wavenumber and frequency of the laser pulse in vacuum are $k_{0}$ and $\omega_{0}$ respectively (inside the material $k=k_{0} n, n$ being the linear refractive index). The first term on the right of Eq. (1) accounts for diffraction, the second for nonlinear refraction $\left(n_{2}\right.$ is the nonlinear refractive index of the material defined by $\left.n(I)=n_{0}+n_{2} I\right)$, the third for group velocity dispersion, while the fourth corresponds to the absorption required for carrier photo-ionization at a rate $W_{P I}$ in a material with a bandgap $U_{i}$. The last term, for sufficiently high free carrier densities, introduces plasma defocusing and absorption associated to an electron plasma with a time dependent density $(\rho)$ and a cross section for inverse bremstrahlung $(\sigma)$.

The solution of Eq.(1) requires considering jointly the temporal evolution of the plasma density and a rate equation including photoionization, avalanche, and plasma relaxation terms $\mathrm{s}^{9,16}$. More detailed formulations for the NLSE including explicitly terms such as Raman, self steepening or space-time focusing can be used, depending on the specific conditions of the interaction problem considered ${ }^{15,17}$. Similarly, simplified versions for negligible photo-generated carrier densities can be used, for instance, to describe the generation of spatial solitons ${ }^{18}$. In all cases the solution of the NLSE requires the use of numerical methods that normally involve a split-step propagation technique or similar procedures ${ }^{9,17}$, for instance a Fourier spectral decomposition in time and a standard Crank-Nicholson scheme in space. A further discussion on the implementation with real-space, spectral or mixed representations is given in Ref. 15. The introduction of transient effects (plasma density evolution including multiple rate equations ${ }^{19}$ or models alike) adds in general difficulties even for surface interactions ${ }^{20}$.

There are situations, however, where it is not necessary to acquire an extremely detailed view of the process in terms of temporal evolution of the carrier density. This is the case, for instance, when trying to make an a priori first guess of the best experimental parameters required for ultrafast sub-surface laser processing of dielectrics ${ }^{21,22}$ using low repetition-rate pulses in the presence of strong aberration $^{23,24}$, prefocal depletion ${ }^{25-27}$ or self-focusing ${ }^{28}$ effects or in standard calculations of spatial soliton formation ${ }^{18}$. Moreover, the exclusive use of the nonlinear absorption term may suffice in several cases to provide an accurate description of the beam reshaping process, also in presence of plasma defocusing, as in the case of beam filamentation in water described by Dubietis and coworkers ${ }^{16}$.

In this work we describe a method for modeling the propagation of laser beams in dielectrics in order to rapidly assess optical beam propagation in the presence of absorptive and refractive nonlinearities by monitoring optical beam reshaping against numerical prediction. We aim to provide such information at a very low computational cost, of the order of minutes, in order to have an effective diagnostic tool for ultrafast laser processing applications. Although the solution of the nonlinear Shroedinger equation is usually not considered a computationally heavy problem, our approach requires a lower computational cost than standard implementations. For this reason, we employ a numerical approach based on a state of the art, generalized fast-Fourier, adaptive evolver (GAFFE: A toolbox for solving evolutionary nonlinear PDEs) ${ }^{29,30}$. While the use of a splitstep method for solving the NLSE is not original in itself, the method here used calibrates itself to a given level of error tolerance and resizes the meshing with respect to the Nyquist limit, yielding a novel and highly efficient approach to compute beam propagation.

In Section I we describe the physical model on which the nonlinear bean propagator is based as well as its limits of validity. Section II provides a brief description of the code as well as several examples of the code performance when calculating nonlinear propagation in the case of spatial soliton shaping and waveguide writing. Simulations are compared to experimental results obtained in soliton generation, as well as real waveguide writing conditions. We show that, from the simple monitoring of the beam shaping it is possible to efficiently extract the effective nonlinear parameters of the material (nonlineal refractive and absorption coefficients) with an accuracy sufficient to predict the beam evolution inside the medium. This approach is particularly valuable for femtosecond laser writing applications. The actual implementation of the propagator is described in Section III (Methods).

\section{Physical Model and Limits of Validity}

The physical model used is based on the solution of the NLSE under simplified interaction conditions:

$2 i k \frac{\partial A}{\partial z}+\left(\frac{\partial^{2}}{\partial^{2} x^{2}}+\frac{\partial^{2}}{\partial^{2} y^{2}}\right) A+\frac{n_{2} k^{2}}{\eta_{0}}|A|^{2} A+i k \beta_{K}\left(\frac{n_{0}}{2 \eta_{0}}\right)^{K-1}|A|^{2 K-2} A=0$ (Eq.2)

where the time-dependent contributions of group velocity dispersion (GVD) and plasma effects have been neglected, and the absorption term associated to photo-ionization has been replaced by an effective nonlinear absorption involving the nonlinear absorption of $K$ photons. Since Eq. (2) neglects the temporal dependencies, the beam propagation can be considered as calculated in a pseudo-stationary regime where a square pulse with constant width $\left(\tau_{p}\right)$ and limited energy propagates changing only its amplitude (i.e. its local intensity $I(x y z))$. This is equivalent to obtaining a snapshot of the energy deposition profile corresponding to the peak power of the beam, when the effects expected from optical nonlinearities are most intense. It is worth noting that Eq.(2) in the absence of nonlinear absorption corresponds to the scalar Helmholtz equation for a Kerr medium under the paraxial approximation ${ }^{31}$.

The first simplification is based on group velocity dispersion effects being important only for very short pulses, highly dispersive materials and/or long propagation distances ${ }^{32}$. To ensure the validity of this assumption before the calculations it is necessary to compute the expected pulse broadening over the considered propagation length in terms of the dispersive length $L_{G V D}=\frac{\tau_{p}^{2}}{2 k^{\prime \prime}}, k^{\prime \prime}=\left.\frac{\partial^{2} k}{\partial \omega^{2}}\right|_{\omega_{0}}$. For instance for a 100 fs FWHM transform-limited pulse centered at $800 \mathrm{~nm}$ and propagating in fused silica $\left(k^{\prime \prime}=36 \mathrm{fs}^{2} / \mathrm{mm}\right), \mathrm{L}_{\mathrm{GVD}}=$ $13.9 \mathrm{~cm}$ and the pulse will broaden approx. $0.5 \%$ after propagating for $1 \mathrm{~cm}$. In general, calculations for propagation distances $d \leq 0.2 \times$ $L_{G V D}$ will not be affected by neglecting GVD and higher order dispersion.

Replacing the absorption term associated to photo-ionization by an effective multi-photon absorption is valid for weak fields (up to $\sim 10^{17} \mathrm{Wm}^{-2}$ depending on the material) ${ }^{14,33}$. For field intensities for which the Keldysh parameter ${ }^{12,34}, \gamma$, is not $\gg 1$, a "local" intensity dependent value for $\beta_{K}$ (derived from the Keldysh model) should be used in the code. As an example Fig. 1(a) shows the intensity computed as a function of the propagation distance for a pulse with an initial energy of $0.25 \mu \mathrm{J}$ in fused silica. The propagation parameters are: wavelength $\lambda=800 \mathrm{~nm}$, pulse duration $\tau=160 \mathrm{fs}$, focusing depth $d=75 \mu \mathrm{m}$ and beam waist $w=1.1 \mu \mathrm{m}$ (inside the material at at low intensity) similar to those in Ref. 9. We used a nonlinear refractive index $n_{2}=2.25 \times 10^{-20} \mathrm{~m}^{2} / \mathrm{W}$ at $800 \mathrm{~nm}^{35-37}$.The effective $\beta_{6}$ is taken as an adjustable parameter in order to reproduce the results of the maximum local intensity $\left(\mathrm{I}_{\max }\right)$ for various pulse energies (up to $1.25 \mu \mathrm{J} /$ pulse) calculated using the Keldysh formulation according to Ref. 32. The so determined $\beta_{6}$ values are shown in Fig. 1(b) along with the corresponding $I_{\max }$ values from Ref. 9.

In Fig. 1(b) the $I_{\text {max }}$ data show a characteristic inflection for a pulse energy of $\sim 0.1 \mu \mathrm{J}\left(\sim 3.0 \times 10^{17} \mathrm{Wm}^{-2}\right)$, indicating the transition from a multi-photon absorption behavior to a tunneling dominated 

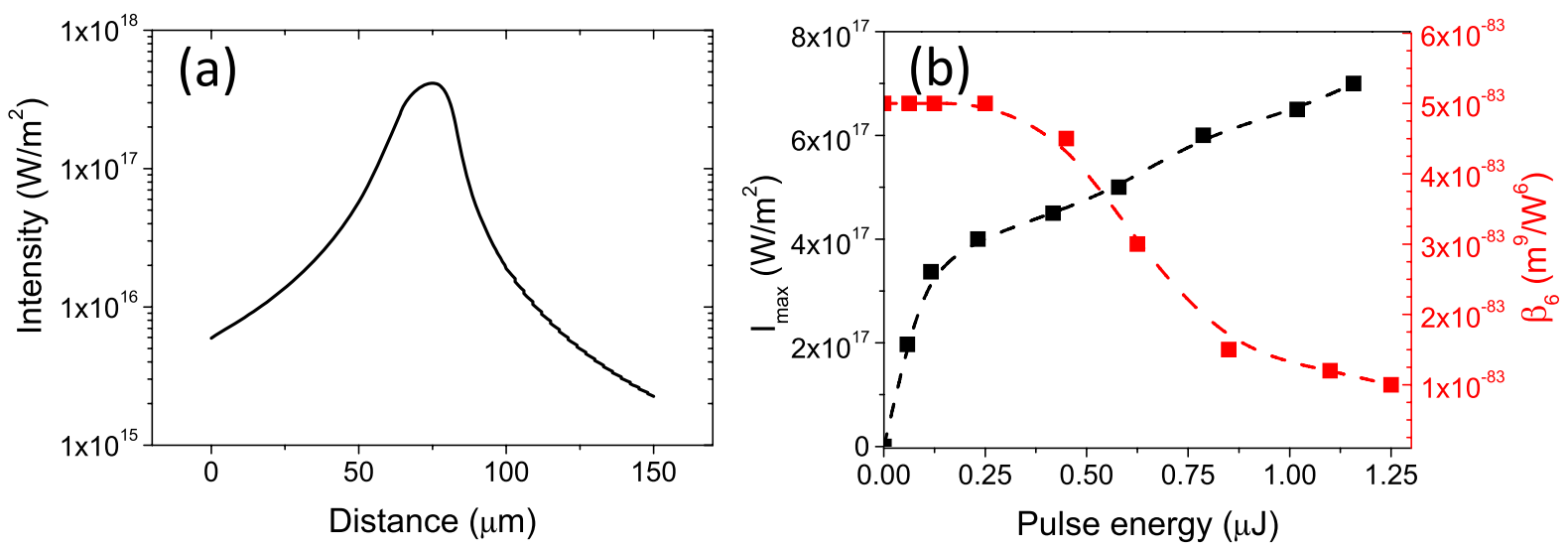

Figure $1 \mid$ (a) Computed intensity as a function of the propagation distance inside the material achieved during the propagation of a focused fs-laser beam in fused silica for a pulse energy of $0.25 \mu \mathrm{J}$. (b) (Black-squares) Computed maximum intensity $\left(\mathrm{I}_{\max }\right.$ ) during propagation as a function of the pulse energy according to Ref. 7 using the Keldysh formulation. (Red squares) Values for the effective intensity-dependent $\beta_{6}$ coefficient derived from adjusting $I_{\max }$ with the code and using $\beta_{6}$ as a fitting parameter. Dashed lines are a guide to the eye.

scenario. The nearly constant effective value of $\beta_{6}\left(5 \times 10^{-83} \mathrm{~m}^{9}\right.$ $\mathrm{W}^{-6}$ ) obtained for the lowest pulse energies indicates that the multi-photon absorption approach can be thus used in our code for local intensities up to $\sim 3.0 \times 10^{17} \mathrm{Wm}^{-2}$ in this case. The indicated $\beta_{6}$ corresponds to a cross section $\sigma_{6}=1.6 \times 10^{-69} \mathrm{~s}^{-1} \mathrm{~cm}^{12} \mathrm{~W}^{-6}$ for the multi-photon ionization rate, which is very close to the one where the Keldysh and multi-photon formulations coincide for low intensities in fused silica according to Ref. $14\left(\sigma_{6}=9.8 \times\right.$ $\left.10^{-70} \mathrm{~s}^{-1} \mathrm{~cm}^{12} \mathrm{~W}^{-6}\right)$. For higher intensities, the calculated effective $\beta_{6}$ decreases, indicating that the rigorous Keldysh formulation is required and that a local intensity-dependent value of $\beta_{6}$ needs be used in the code when computing the propagation.

Equation (2) neglects transient plasma defocusing and absorption effects. As indicated above, it is well known that using just the nonlinear absorption term ${ }^{16}$ may often yield results sufficiently accurate to calculate the evolution of the beam shape/intensity upon propagation. In this respect, the intensity threshold for plasma self-defocusing effects to become important is not sharply defined. As thoroughly discussed by Brodeur and coworkers ${ }^{38}$ the compensation of nonlinear self-focusing by an electron plasma requires carrier generation rates of the order of $10^{-18} \mathrm{~cm}^{-3} \mathrm{fs}^{-1}$. The exact value depends on the material bandgap, the nonlinear refractive index and the laser wavelength. Carrier generation for a 100 fs laser pulse leads to limiting intensities $\left(I_{\text {stop }}\right)$ for compensating the Kerr effect in the $10^{-16}$ $10^{-17} \mathrm{Wm}^{-2}$ range and electronic densities in the $10^{17}-10^{19} \mathrm{~cm}^{-3}$ range in water. This limits the validity of the results derived from Eq. (2) to local intensities typically below $\sim 10^{17} \mathrm{Wm}^{-25,38}$ or carrier generation rates typically below $\sim 10^{18} \mathrm{~cm}^{-3} \mathrm{fs}^{-1}$, as in those cases where the multi-photon absorption approach is used instead of the rigorous Keldysh formulation. Similarly, the presence of avalanche effects limits the use of Eq.(2) when the local intensity of the pulse multiplied by its duration exceeds values typically of the order of $1 \mathrm{~J} /$ $\mathrm{cm}^{2}$, as shown in silica by Rethfeld for avalanche dominated processes, according to the multiple rate equations (MRE) model ${ }^{39}$. Using as validity limits a maximum local intensity $I_{\max }<$ $\left(\sim 10^{17} \mathrm{Wm}^{-2}\right)$ and a product $I_{l o c} \times \tau_{p}<\sim 1 \mathrm{~J} / \mathrm{cm}^{2}$, depending on the material, should enable obtaining results in terms of energy deposition sufficiently close to the experimental situation. We will see that for the case of elliptical beams, the peculiarities of the nonlinear propagation allow overcoming this "conservative" local intensity limit still providing a very useful picture of the spatial distribution of energy deposition.

\section{Results and discussion}

In brief, in order to solve Eq.2 a split-step Fourier method is used with a library of optimized functions developed by E. Grace ${ }^{30}$ to speed up the calculation time. This library contains routines to dynamically resize the dimensions (in the $x$ and $y$ axis) and number of points of the mesh that represents the field we propagate in the sample. This is done in order to ensure that we have a full representation of the propagating field using a minimum number of points. The step-size taken in the propagation direction of the beam ( $z$ axis) is also optimized so as to use the largest possible step within a certain error tolerance, given by comparing the propagation of the same beam for two different step lengths. This allows obtaining results in a very short time (typically a few tens of seconds) using a laptop computer and testing a large number of parameters in a short time. A detailed description of the implementation of the code is given in the Methods Section below.

In this section we analyze the results of the numerical model in several cases where the effects of nonlinear refraction and absorption are important in defining the energy deposition or propagation spatial profile of a high intensity beam. The first example refers to the generation of spatial solitons in a strongly nonlinear Kerr material where catastrophic collapse is avoided by multi-photon absorption. In this case, the model given in Eq.(2) can be considered almost a rigorous description of the problem, where only the temporal effects are neglected. The other examples refer to situations where neglecting transient plasma effects can affect the overall validity of the model and thus the results have to be considered as only approximate. Nevertheless we will show that, for Gaussian elliptical beams in space, the sensitivity of the spatial energy deposition profile to relatively small changes in $n_{2}$ and $\beta_{K}$ is sufficient to perform reasonable estimates of both parameters from a comparison with images of the irradiated material in the focal region. This enables using the nonlinear propagator for a priori optimization of the experimental conditions for sub-surface processing with a very small computational cost. This latter feature is of special interest for fs-laser waveguide writing and other sub-surface nonlinear laser processes. Being able to inexpensively model the physical process more quickly than doing the experiment opens up opportunities for finding material parameters that could be beneficial for a given application.

Propagation for negligible transient plasma effects: Soliton shaping. Following the description by Pasquazi and coworkers in Ref. 18, we simulated the propagation of a 25 ps laser pulse at $820 \mathrm{~nm}$, focused at the surface of a $25 \mathrm{Nb}_{2} \mathrm{O}_{5}-25 \mathrm{PbO}-50 \mathrm{GeO}_{2}$ mol.\% (NPG) heavy metal oxide glass and leading to the formation of a spatial soliton with the aid of three-photon absorption. In this case, picosecond excitation allows us excluding the presence of significant GVD and plasma formation effects, making fully applicable the description of the process by Eq.(2). 
In order to determine the nonlinear refractive index $\left(n_{2}\right)$ and the three-photon absorption coefficient $\left(\beta_{3}\right)$ of the material, several propagation simulations using different values of $n_{2}$ and $\beta_{3}$ were performed in order to reproduce the experimentally observed intensity profile at the output facet of the sample after propagation. In the experiments the beam $(820 \mathrm{~nm}, 25 \mathrm{ps}$ duration (FWHM)) was focused at the input facet with a $3.5 \mathrm{~cm}$ focal length lens to a waist of $11 \mu \mathrm{m}$ and propagated over a distance of $5.7 \mathrm{~mm}$. The formation of a fundamental soliton was observed at a pulse energy of $2.8 \mu \mathrm{J}^{18,40}$.

The initial simulations were carried out with a few fixed values of $n_{2}$ in the $10^{-19}-10^{-18} \mathrm{~m}^{2} / \mathrm{W}$ interval (as determined by degenerate four wave mixing ${ }^{41}$ ), using $\beta_{3}$ as the fitting parameter in order to get a fundamental soliton at a pulse energy of $2.8 \mu \mathrm{J}$. Each propagation simulation typically lasted some $30-40$ seconds on a normal laptop computer enabling to perform, over a relatively small time, a complete set of iterations varying $\beta_{3}$ for a given $n_{2}$. The validity of the optimal values found for $\mathrm{n}_{2}$ and $\beta_{3}$ was then tested by simulating the propagation for other pulse energies and comparing the result to experimentally obtained images. The results are shown in Fig. 2 where both experimental and calculated values of the intensity profile at the sample output facet are shown versus pulse energy. The optimal values determined from the simulation were $n_{2}=5.5 \times$ $10^{-19} \mathrm{~m}^{2} / \mathrm{W}$ and $\beta_{3}=5.5 \times 10^{-28} \mathrm{~m}^{3} / \mathrm{W}^{2}$. The values reported in Ref. 18 are $n_{2}=5.5 \times 10^{-19} \mathrm{~m}^{2} / \mathrm{W}$ and $\beta_{3}=3.0 \times 10^{-28} \mathrm{~m}^{3} / \mathrm{W}^{2}$, and were extracted by the complete fitting of the experimental results comprising an independent measurement of the nonlinear transmission of the glass and accounting for the actual temporal shape of the Gaussian pulse. The temporal dependence, even when GVD is neglected, is required for a more accurate fitting of the pulse selffocusing considering that the measured images were obtained by integration of the pulse profile.

The tails of the pulse undergo a less pronounced self-focusing than the peak. Hence, for the same peak power, a temporally Gaussian pulse produces a soliton beam that appears larger than the one generated by a square pulse. For this reason our model here requires a larger nonlinear absorption coefficient to counteract the self-focusing effect, as compared with what experienced by the pulse during experiments. Nevertheless, the computation time for the propagation of a single temporally Gaussian pulse is more than 20 times longer. With this approach we obtain the same $n_{2}$ and an upper estimate of $\beta_{3}$ of the same order of magnitude but with a computation time (minutes) compatible with the real time evaluation of the material parameters by the direct monitoring of beam reshaping at the output.

Propagation for non-negligible transient plasma effects: Sensitivity of the energy deposition profile to the input beam shape. The utility of the developed propagator in situations where photo-generated carrier effects cannot be neglected a priori is given by the strong sensitivity of the spatial intensity distribution in the material to the actual shape of the input beam. This is illustrated in Fig. 3 showing the calculated spatial intensity distribution in the focal region for two beams (Gaussian circular and elliptical, respectively) with the same initial pulse energy and duration, focused at the same depth (with the same optics) for different values of $n_{2}$ and $\beta_{6}$ and realistic waveguide writing conditions. In the figure, all the simulations correspond to an input beam of diameter $7 \mathrm{~mm}$ (1/ $\mathrm{e}^{2}$ intensity), $100 \mathrm{fs}$ (FWHM) duration, $7.5 \mu \mathrm{J}$ pulse energy at $800 \mathrm{~nm}$, focused with a $20 \mathrm{~mm}$ focal length lens (beam NA = 0.17) $500 \mu \mathrm{m}$ below the surface. The Gaussian elliptical beam has its long axis along $X$ with an aspect ratio $R_{x} / R_{y}=20$, and propagates along $Z$. The conditions in the simulations are similar to the typical experimental parameters used for fs-laser waveguide writing at low repetition rates with elliptical beam shaping ${ }^{26}$ at moderate writing depths ${ }^{42}$ in fused silica (a nominal six photon process at $800 \mathrm{~nm}$ ).

In all cases, the simulations including nonlinearities $\left(n_{2} \neq 0, \beta_{6} \neq\right.$ 0 ) show maximum local intensities in the $1 \times 10^{17}-5 \times 10^{17} \mathrm{Wm}^{-2}$ range, close to and above the theoretical validity limit of Eq.(2). The corresponding maximum electronic densities and carrier generation rates have been estimated by computing the local nonlinear loss and converting it to the corresponding energy assuming a Gaussian temporal profile and a 6-photon absorption process. This leads to carrier generation rates of $6.0 \times 10^{18}-2.4 \times{ }^{19} \mathrm{~cm}^{-3} \mathrm{fs}^{-1}$, above the threshold initially taken for neglecting plasma self defocusing. Still, it is worth comparing the evolution of the modeled intensity cross sections with and without slit shaping for various nonlinear coefficients. First, in the focal region the transversal energy spread associated to the elliptical beam makes the differences in the spatial intensity distribution for various values of $n_{2}$ and $\beta_{6}$ much more visible than in a circular beam, despite that the local maximum intensities are higher in the second case. It is also clear that changes in $n_{2}$ over one order of magnitude have a much stronger impact than changes in $\beta_{6}$ in the intensity distribution in the focal region. These latter may be hardly distinguished in terms of shape for the circular beam (compare Fig. 3(b) and (c)) unlike the case of the elliptical beam, in which the energy deposition in the pre-focal region enables to clearly reveal changes in $\beta_{6}$ within one order of magnitude (see Fig. 3 (f) and (g)). Similar simulations as in Fig. 3 show that analogous features can be observed for different numbers of absorbed photons, $K \geq 2$, although the sensitivity of the intensity cross section shape to changes in $\beta_{K}$ is higher the lower the $K$ value is. As a general trend, the computed intensity profiles permit to distinguish changes in $n_{2}$ and $\beta_{K}$
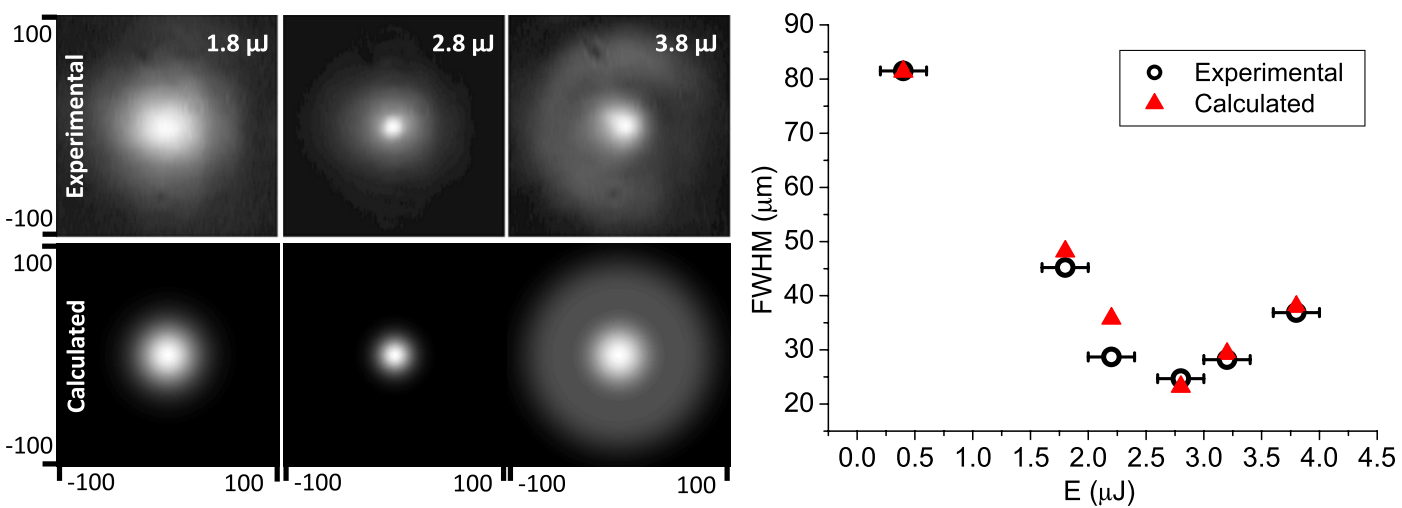

Figure 2 (left) Experimental and calculated images of the transverse beam intensity distribution at the output exit of the NPG glass for different pulse energy values. (right) Experimental (from Ref. 40) and calculated beam waist (FWHM) at the exit facet of the sample as a function of the input pulse energy. The error bars indicate the error in the experimental pulse energy determination. The scales are in $\mu \mathrm{m}$. The coefficients used in the simulations are $n_{2}=5.5 \times 10^{-19} \mathrm{~m}^{2} / \mathrm{W}$ and $\beta_{3}=5.5 \times 10^{-28} \mathrm{~m}^{3} / \mathrm{W}^{2}$. 


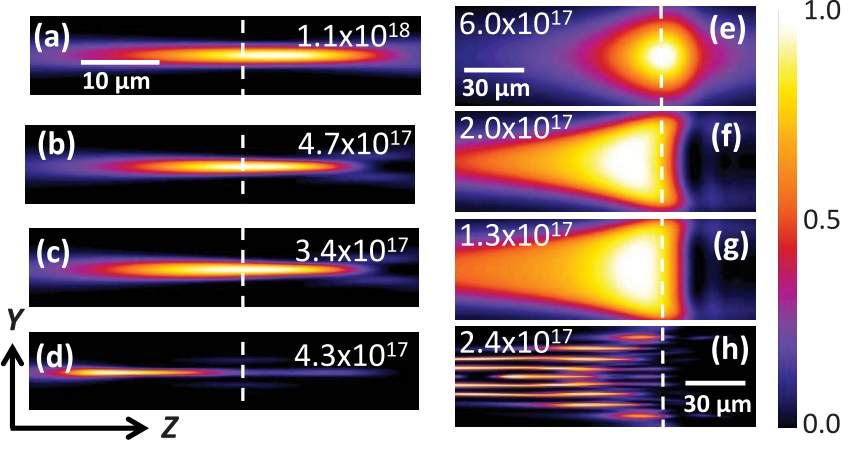

Figure 3 Simulated intensity $Y-Z$ cross sections $(x=0)$ in the focal region for various $n_{2}$ and $\beta_{6}$, for a $100 \mathrm{fs}, 7.5 \mu \mathrm{J}$ pulse, focused $500 \mu \mathrm{m}$ underneath the surface with a $20 \mathrm{~mm}$ focal length lens. (a-d) Correspond to a Gaussian circular beam $\left(R_{x}=R_{y}=3.5 \mathrm{~mm}\left(1 / \mathrm{e}^{2}\right)\right)$. (e-f) Correspond to a Gaussian elliptical beam $\left(R_{x}=3.5, R_{y}=0.175 \mathrm{~mm}(1 / \mathrm{e} 2)\right)$. The nonlinear parameters in the simulations are $n_{2}=0, \beta_{6}=0(\mathrm{a}, \mathrm{e}), n_{2}=$ $10^{-20} \mathrm{~m}^{2} \mathrm{~W}^{-1}, \beta_{6}=5 \times 10^{-84} \mathrm{~m}^{9} \mathrm{~W}^{-5}(\mathrm{~b}), n_{2}=10^{-20} \mathrm{~m}^{2} \mathrm{~W}^{-1}, \beta_{6}=5 \times$ $10^{-83} \mathrm{~m}^{9} \mathrm{~W}^{-5}$ (c), $n_{2}=10^{-19} \mathrm{~m}^{2} \mathrm{~W}^{-1}, \beta_{6}=5 \times 10^{-84} \mathrm{~m}^{9} \mathrm{~W}^{-5}(\mathrm{~d}), n_{2}=$ $10^{-20} \mathrm{~m}^{2} \mathrm{~W}^{-1}, \beta_{6}=5 \times 10^{-82} \mathrm{~m}^{9} \mathrm{~W}^{-5}(\mathrm{f}), n_{2}=10^{-20} \mathrm{~m}^{2} \mathrm{~W}^{-1}, \beta_{6}=5 \times$ $10^{-81} \mathrm{~m}^{9} \mathrm{~W}^{-5}(\mathrm{~g}), n_{2}=10^{-19} \mathrm{~m}^{2} \mathrm{~W}^{-1}, \beta_{6}=5 \times 10^{-82} \mathrm{~m}^{9} \mathrm{~W}^{-5}$ (h). All images were normalized to the maximum power density indicated by the legend in each image in $\mathrm{Wm}^{-2}$. The spatial scales are the same for $(\mathrm{a}-\mathrm{d})$ and $(\mathrm{e}-\mathrm{h})$, respectively. The beam incidence is along $\mathrm{Z}$. The white dashed indicates the linear focus position.

within a factor of two and within one order of magnitude, respectively, for elliptical beams with an aspect ratio $R_{x} / R_{y} \geq$ $\sim 5$.

Propagation for non-negligible transient plasma effects: Determination of nonlinear coefficients $\left(n_{2}, \beta_{K}\right)$ from imaging experiments. These peculiarities of the nonlinear propagation of elliptical beams can be exploited to estimate the material nonlinear coefficients $\left(n_{2}, \beta_{K}\right)$ from post-mortem trans-illumination images of the focal region or plasma emission images ${ }^{21}$. Figure $4(\mathrm{a}-\mathrm{d})$ shows several cross sectional images of fs-laser written waveguides in fused silica using the slit shaping technique ${ }^{43}$ for various slit-widths, pulse energies and writing depths as described in Ref. 42, along with the corresponding propagation modes at $633 \mathrm{~nm}$. The writing conditions correspond to a linearly polarized input beam of diameter $7.4 \mathrm{~mm}$ (1/ $\mathrm{e}^{2}$ intensity), $100 \mathrm{fs}$ (FWHM) pulse duration at $800 \mathrm{~nm}$, focused with a $20 \mathrm{~mm}$ focal length lens below the surface after being shaped with a variable slit. The laser repetition rate was $1 \mathrm{kHz}$ and the sample scanning speed $100 \mu \mathrm{m} / \mathrm{s}$. The slight asymmetry in the experimental results with respect to the symmetry plane $(X-Z)$ is most likely due to a slight tilt on the input beam.

The experimental images clearly show the combined effect of slit shaping, nonlinear refraction and increased propagation depths. For the shallowest depth and smaller energy $(0.75 \mathrm{~mm}, 4 \mu \mathrm{J}$, Fig. $4(\mathrm{a}))$ the accumulated nonlinear phase shift is still relatively small to create a large distortion of the energy deposition profile with respect to the linear case. It is worth noting that, as indicated in Ref. 42, the use of a relatively small NA $(\sim 0.19)$ and slit shaping strongly diminishes spherical aberration, leading to a relatively small transformed region along the beam propagation axis. As the pulse energy and the processing depth increase, nonlinear self-focusing, and spherical aberration distort the focal volume, leading to a characteristic arrow-head shape (Fig. 4(b)). The angle and length of the arrow-head is given by the combination of pulse energy, processing depth and slit width, as shown in Fig. (4b-d). This is rather apparent for the deepest structure shown (writing depth $4.35 \mathrm{~mm}$ ) for which spherical aberration effects are clearly appreciable.
In order to model the observed structural changes, the effect of the slit is replaced by an elliptically shaped beam with the corresponding aspect ratio. The propagation calculations were performed using an intensity independent $\beta_{6}$ coefficient of $5 \times 10^{-83} \mathrm{~m}^{9} \mathrm{~W}^{-6}$ (valid for maximum local intensities below $\sim 4 \times 10^{17} \mathrm{Wm}^{-2}$ (see Section II)) and using $n_{2}$ as an adjustable parameter in order to reproduce the characteristic shape of the transformed region. To this extent we used the spatial distribution of the nonlinear absorbed energy, as shown in the lower row of Fig. 4 where the corresponding normalized values were discretized to ease the comparison with the shape of the transformed region. The same value, $n_{2}=0.75 \pm 0.25 \times 10^{-20} \mathrm{~m}^{2} / \mathrm{W}$, was determined from several structures similar to those shown in Fig. 4. This value is substantially smaller than the typically reported values for the nonlinear refractive index of un-irradiated fused silica at $800 \mathrm{~nm}\left(2.0-2.5 \times 10^{-20} \mathrm{~m}^{2} / \mathrm{W}^{9,37}\right.$.

This apparently surprising result is perfectly consistent with the observations reported by Blömer and coworkers ${ }^{44}$ on the nonlinear refractive index of waveguides written in fused silica at $\mathrm{KHz}$ repetition rates. Their estimates, based on self modulation measurements during the propagation of fs-laser pulses in the written waveguides, show a substantial decrease of $n_{2}$ in the laser irradiated material, up to a factor $\sim 4$ for the maximum dose analyzed. In our case, the estimated ratio $n_{\text {2irradiated }} / n_{2 \text { non-irradiated }} \sim 3$ is very close and consistent with the lower dose used. Interestingly, our simulation is a clear indication that the modification of the nonlinear refractive index of the material occurred during the interaction (it is not an aftereffect) as it conditioned the energy deposition process during the writing process.

It is also worth noting that these $n_{2}$ estimates correspond to a situation in which the achieved maximum local intensities are above the conservative limit at which plasma self-focusing can be neglected $\left(10^{17} \mathrm{Wm}^{-2}\right.$, see Section I). In fact the maximum carrier generation rates and carrier densities calculated from the simulations in Fig. 4 are in the $5 \times 10^{18} \mathrm{~cm}^{-3} \mathrm{fs}^{-1}$, and $8 \times 10^{20} \mathrm{~cm}^{-3}$ ranges, respectively. This latter value is perfectly consistent with experimental data from maximum plasma densities measured in waveguide writing by fsresolved microscopy ${ }^{26}$. This pinpoints that, as expected from the comparison of simulated absorbed energy cross sections and experimental waveguide cross section images, the calculations provide reliable estimates of the energy deposition profile even above the plasma self-defocusing threshold. The actual reason for such a remarkable outcome is not totally clear: on the one hand, the highest plasma densities are reached near-before the focal region (or well before it depending on the amount of accumulated nonlinear phase-shift) where the beam is strongly depleted by nonlinear absorption. As a consequence, plasma self-defocusing effects may be strongly reduced. On the other hand, the plasma build-up speed could -to some extent- soften plasma self-defocusing effects while nonlinear refractive index changes (like the ones we observe upon irradiation) dynamically affect the carrier generation rate. It should also be noted that avalanche process is delayed and thus cannot compensate the Kerr effect when the pulses are sufficiently short. In any case, the results derived from the image analysis show that, owing to the sensitivity of the focal volume of an elliptical beam to nonlinear phenomena during propagation, the presented approach is quite reliable in determining the effective nonlinear coefficients under actual writing conditions. In general, as also pointed out in Ref 16, this study suggests that the solution of the nonlinear Schrödinger equation with nonlinear absorption alone may suffice to capture the dynamics of beam reshaping in a number of cases beyond the plasma-free regime. This seems to be the case for elliptical beams, partly due also to the peculiarities of their propagation in presence of absorptive and refractive nonlinearities.

This approach for the estimation of nonlinear coefficients is similarly valid for processes involving a lower number of photons in the nonlinear absorption process, as shown in Fig. 5. The figure shows 

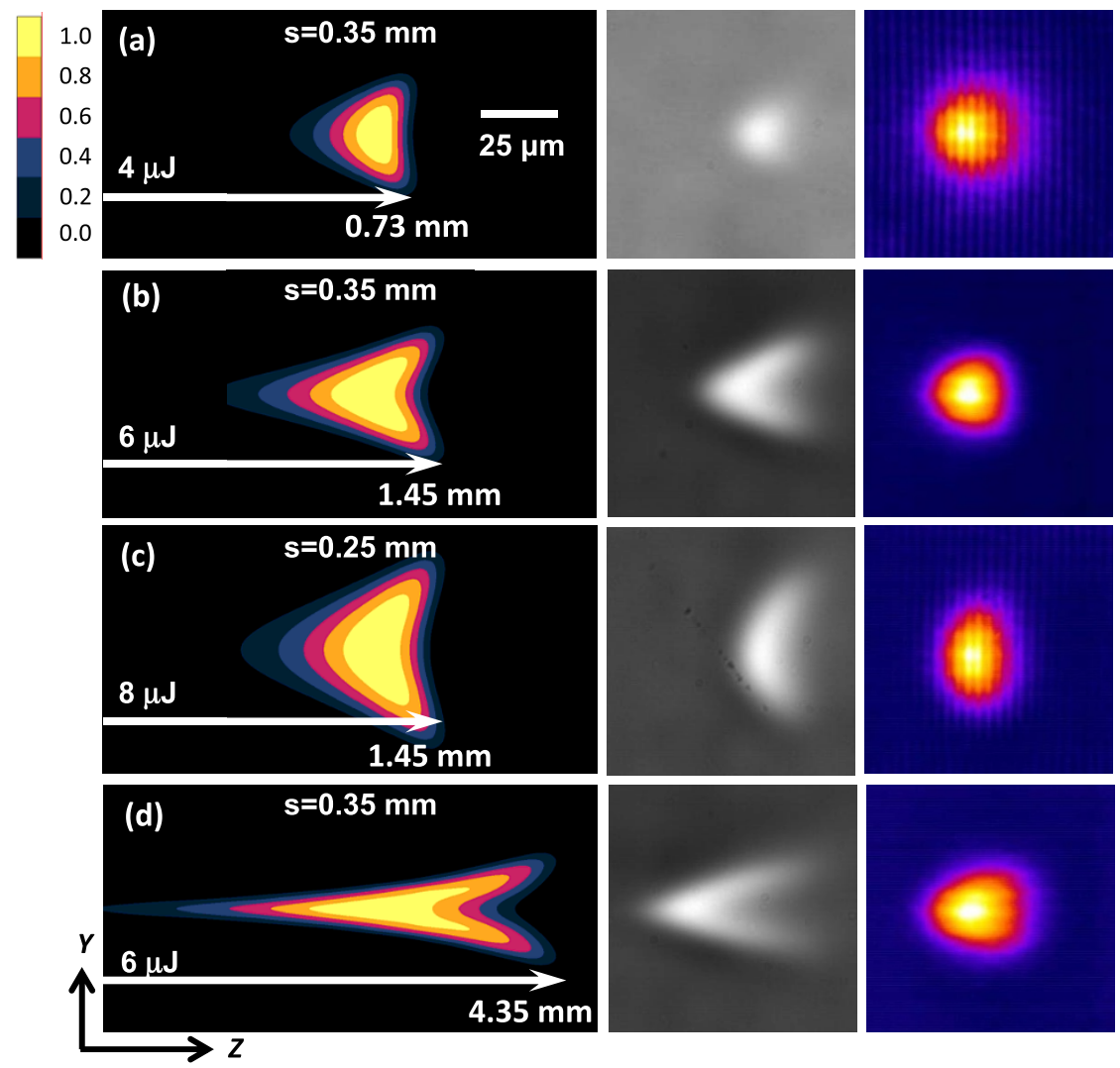

Figure $4 \mid$ Central column, cross sectional transillumination images ( $\mathrm{Y}-\mathrm{Z}$ plane) of waveguides written in fused silica using slit shaping at the indicated energies $(\mu \mathrm{J} /$ pulse), slit widths and processing depths. Beam incidence is from the left of the images. The corresponding propagated modes at $633 \mathrm{~nm}$ are shown in the column to the right. The left column shows the corresponding normalized cross sectional images of the calculated distribution of absorbed energy along the $\mathrm{Y}-\mathrm{Z}$ plane $(\mathrm{x}=0)$. The arrows indicate the position of the linear focus at the indicated processing depth. The absorbed energy

distributions have been normalized to the maximum (1.0) and then discretized in 0.2 steps to facilitate the comparison with the experimental images. The calculated absorbed energies are $1.9(\mathrm{a}), 3.8(\mathrm{~b}), 4.9$ (c) and $3.9 \mu \mathrm{J}(\mathrm{d})$, respectively. The simulations were performed using $n_{2}=0.75 \times 10^{-20} \mathrm{~m}^{2} / \mathrm{W}, \beta_{6}=$ $5 \times 10^{-83} \mathrm{~m}^{9} \mathrm{~W}^{-5}$. In all cases the maximum local intensity achieved is $\sim 2 \times 10^{17} \mathrm{Wm}^{-2}$.

two cross sectional images (Fig. 5(a), 5(b)) of waveguides produced in a commercial phosphate glass by fs laser writing at $1 \mathrm{kHz}$ repetition rate with a sample scanning speed of $100 \mu \mathrm{m} / \mathrm{s}$, as reported in Ref. 21 using pulses of different duration and polarization. The guided modes of both structures at $1550 \mathrm{~nm}$ are included as well. The input beam diameter was $7.0 \mathrm{~mm}$ (1/ $\mathrm{e}^{2}$ intensity), shaped with a $0.35 \mathrm{~mm}$ width slit $\left(R_{y} / R_{x}=1 / 20\right.$ aspect ratio $)$ and focused $1.56 \mathrm{~mm}$ below the surface with a $20 \mathrm{~mm}$-focal length microscope objective.

In this case, the shape of the high energy waveguide (Fig. 5.(a)), written with linear polarization, 100 fs laser pulses was first fitted using the nonlinear refractive index of the non-irradiated phosphate glass $^{26}\left(5.85 \times 10^{-20} \mathrm{~m}^{2} \mathrm{~W}^{-1}\right)$ as the starting parameter. Afterwards, optimal values for both $\mathrm{n}_{2}$ and $\beta_{3}$ for linear polarization were determined through an iterative process, leading to $n_{2 L}=1.1 \times$ $10^{-20} \mathrm{~m}^{2} \mathrm{~W}^{-1}$ and $\beta_{3 L}=4.0 \times 10^{-30} \mathrm{~m}^{3} \mathrm{~W}^{-2}$. Consistently with our previous observation in the case of fused silica, the nonlinear refractive index of the irradiated material considerably decreases (by a factor close to 6) with respect to that of the non-irradiated one $e^{45}$. The simulations of the low energy waveguide, written with circular polarization, and a pulse duration of $250 \mathrm{fs}$ (Fig. 5.(b)) were then performed decreasing $\mathrm{n}_{2 \mathrm{~L}}$ by a factor of $1.5\left(n_{2 \mathrm{~L}} / \mathrm{n}_{2 C}=1.5\right)$, corresponding to the ratio of the nonlinear refractive index for linear and circular polarizations in isotropic materials ${ }^{26,35}$. Interestingly, we observe that the change of the irradiation conditions (polarization and pulse duration) not only affects the nonlinear refractive index value but also the nonlinear absorption coefficient. The optimal value of $\beta_{3}$ estimated for circular polarization is $\beta_{3 C}=1.0 \times 10^{-30} \mathrm{~m}^{3} \mathrm{~W}^{-2}$, which is a factor of $\sim 4$ smaller than $\beta_{3 L}$. This diminution of the nonlinear absorption for circular polarization has been experimentally reported by Temnov and coworkers ${ }^{45}$ in crystalline and amorphous dielectrics (in fused silica a similar diminution is observed). The transition from circular-polarization dominance to linear polarization dominance in the nonlinear absorption cross section is theoretically predicted ${ }^{46}$ for $K \sim 4$, although we observe this effect for $\mathrm{K}=3$.

As in the case of fused silica (Fig. 4), the corresponding estimated maximum carrier generation rates and densities $(\sim 6 \times$ $10^{18} \mathrm{~cm}^{-3} \mathrm{fs}^{-1}$ and $\sim 10^{21} \mathrm{~cm}^{-3}$ ) are above the plasma self-defocusing threshold and consistent with experimental measurements ${ }^{26}$, supporting the viability of the approach in a wide variety of processing conditions. The comparisons in Fig. 5 between the calculated incident intensity and absorbed energy cross sections, and the experimental transillumination images of both waveguides suggest that along three photon absorption (responsible for the formation of the refractive index increased, white contrasted region) there is an additional nonlinear energy deposition mechanism. This causes the formation of the dark contrasted zones before and after the focal region, most likely due to the generation of defects. This is valuable information that has to be considered to further improve the performance of the waveguides.

The feasibility of accessing the nonlinear coefficients of a dielectric, even within a resolution of one order of magnitude, based on post-mortem or plasma emission images of the focal region appears thus as an appealing use of the propagator, especially considering its low computational cost and the inherent difficulties in the precise experimental determination of $n_{2}$ and $\beta_{K}$. 

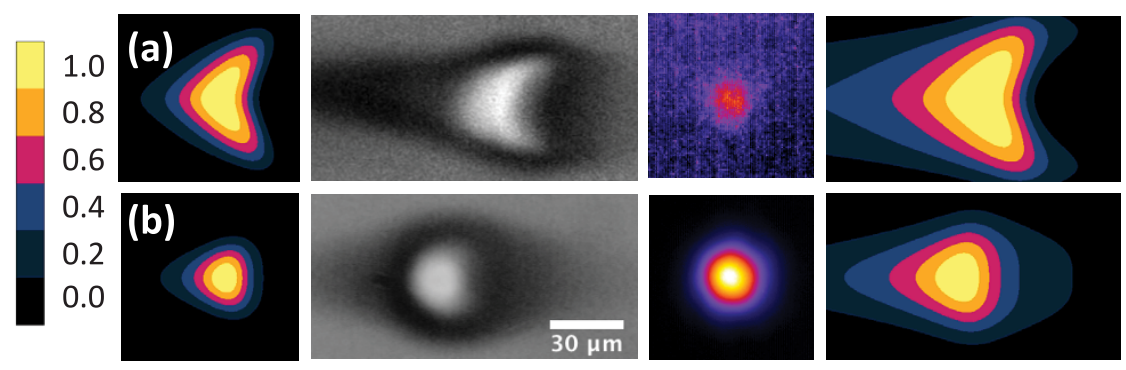

Figure $5 \mid$ (Second colum from left) Cross sectional transillumination images (Y-Z plane) of waveguides written in phosphate glass using slit shaping at energies and pulse durations of $9.6 \mu \mathrm{J} /$ pulse, $100 \mathrm{fs}$ (a) and $4.0 \mu \mathrm{J} / \mathrm{pulse}, 250 \mathrm{fs}(\mathrm{b})$, respectively, for a slit width of $0.35 \mathrm{~mm}$, at a processing depth of $1.56 \mathrm{~mm}$. The polarization of the writing beam is linear in (a) and circular in (b). Beam incidence is from the left of the images. (Left column) Corresponding normalized cross sectional images of the calculated absorbed energy distribution along the $\mathrm{Y}-\mathrm{Z}$ plane $(\mathrm{x}=0)$ for the same processing parameters. The corresponding guided modes at $1550 \mathrm{~nm}$ are shown in the third column from the left. (Right column) Normalized calculated incident intensity cross section. The calculated absorbed energies are 8.5 (a) and $1.9 \mu \mathrm{J}(\mathrm{b})$ while the maximum intensities are $1.5 \times 10^{17}$ and $2.9 \times 10^{17} \mathrm{Wm}^{-2}$, respectively. The simulations were performed using $n_{2}=1.1 \times 10^{-20}$ (a) and $0.72 \times 10^{-20}(\mathrm{~b}) \mathrm{m}^{2} \mathrm{~W}^{-1}$, and $\beta_{3}=4.0 \times 10^{-30}$ (a) and $1.0 \times 10^{-30} \mathrm{~m}^{3} \mathrm{~W}^{-2}(\mathrm{~b})$.

\section{Conclusion}

A method for modeling the propagation of laser beams in dielectrics in the presence of absorptive and refractive nonlinearities has been implemented. The method is based on the use of a generalized fastFourier evolver to solve the nonlinear Shrödinger equation under simplified interaction conditions, with computing times of just tens of seconds. The propagator provides excellent results for propagated power densities at which free carrier generation effects can be neglected. The peculiarities of the nonlinear propagation of elliptical beams enable to use to code in situations where plasma self-defocusing effects are expected to be relevant. In such cases, the code still provides an instantaneous picture of the spatial distribution of the energy deposition in the material sufficiently realistic to perform reasonably accurate estimates of the effective nonlinear refractive index and the nonlinear absorption coefficient based on trans-illumination images of the irradiated region. These estimates can in turn be used for a priori refining the processing conditions of dielectrics with ultra-short laser pulses at low repetition rates in waveguide writing and other applications.

\section{Methods}

Figure 6 shows a flow diagram of the code. For the initial beam, the field, both in the real and Fourier spaces, is represented by a 2-D matrix containing both the corresponding field scalar amplitudes and additional null field values surrounding the field distribution. The representation matrix size, density and beam to space ratio is defined in the real and Fourier spaces before starting the propagation code. The spatial profile of the beam is initially defined in the form of a spatially (circular or elliptical) Gaussian intensity distribution with a (pre-defined) arbitrary wavefront. The potential use of arbitrary wavefronts allows introducing different types of aberrations in the wavefront in order to tailor the spatial profile of the region where energy is deposited, like in the case of using a spatial light modulator for spatial beam shaping ${ }^{47}$. This particular application is however beyond the scope of this manuscript. The beam is then linearly propagated in air and focused by an optically thin lens to reach the air-dielectric interface. The spherical aberration effect associated to the propagation through the interface is then impressed in the beam wavefront following the Fresnel reflection. After this stage, the "health" of the beam is checked in order to ensure the absence of aliasing effects which would render inaccurate results. This is done by measuring the energy fraction of the beam in the outermost regions of the representation matrices (real and Fourier spaces). If the value is above a pre-fixed tolerance limit, the representation matrices have to be redefined until aliasing falls within the specified tolerance. If the field is not aliased, the propagation inside the material begins.

The propagation is performed in small split steps along the propagation direction. For a propagation length $(\Delta z)$, the linear propagation over $\Delta z / 2$ is first computed in the Fourier space. Then the nonlinear propagation (non-linear absorption and refraction) is calculated over the whole step $\Delta z$ in the real and Fourier spaces using as input the previously calculated field amplitude $\mathrm{A}(x, y, z+\Delta z / 2)$. Finally, the remaining linear propagation step over $\Delta z / 2$ is calculated. We can see this as applying the nonlinear propagation over a field that is averaged over the propagation step. Two tests are performed before the computed field amplitude $A(x, y, z+\Delta z)$ is stored and the next is step calculated. First, it is checked if the step size used $\Delta z$ was correct. For this purpose, the field amplitude $A(z-\Delta z)$ is propagated over a step $2 \Delta z$ and compared to the just calculated $A(z+\Delta z)$ (except for the first step). If the difference between both fields is lower than a given tolerance value, the propagation field is taken as correct. If the difference is much lower than the tolerance value, the code increases $\Delta z$ for the next calculation step to reduce the computation cost. If the difference is above tolerance, $\Delta z$ is reduced and the current propagation step is repeated. The second test computes the fraction of energy in the outermost region of the representation matrices. If the field is aliased in the real or in Fourier spaces, the density of the representation matrix in the non-aliased space is increased and the propagation step repeated in the outermost region the representation matrices is sufficiently low, the code reduces the matrix density to reduce the calculation time. It can happen that the new $\Delta z$ is too small and required the time to complete the propagation gets unaffordable. In that case, the calculation must be restarted with new initial field

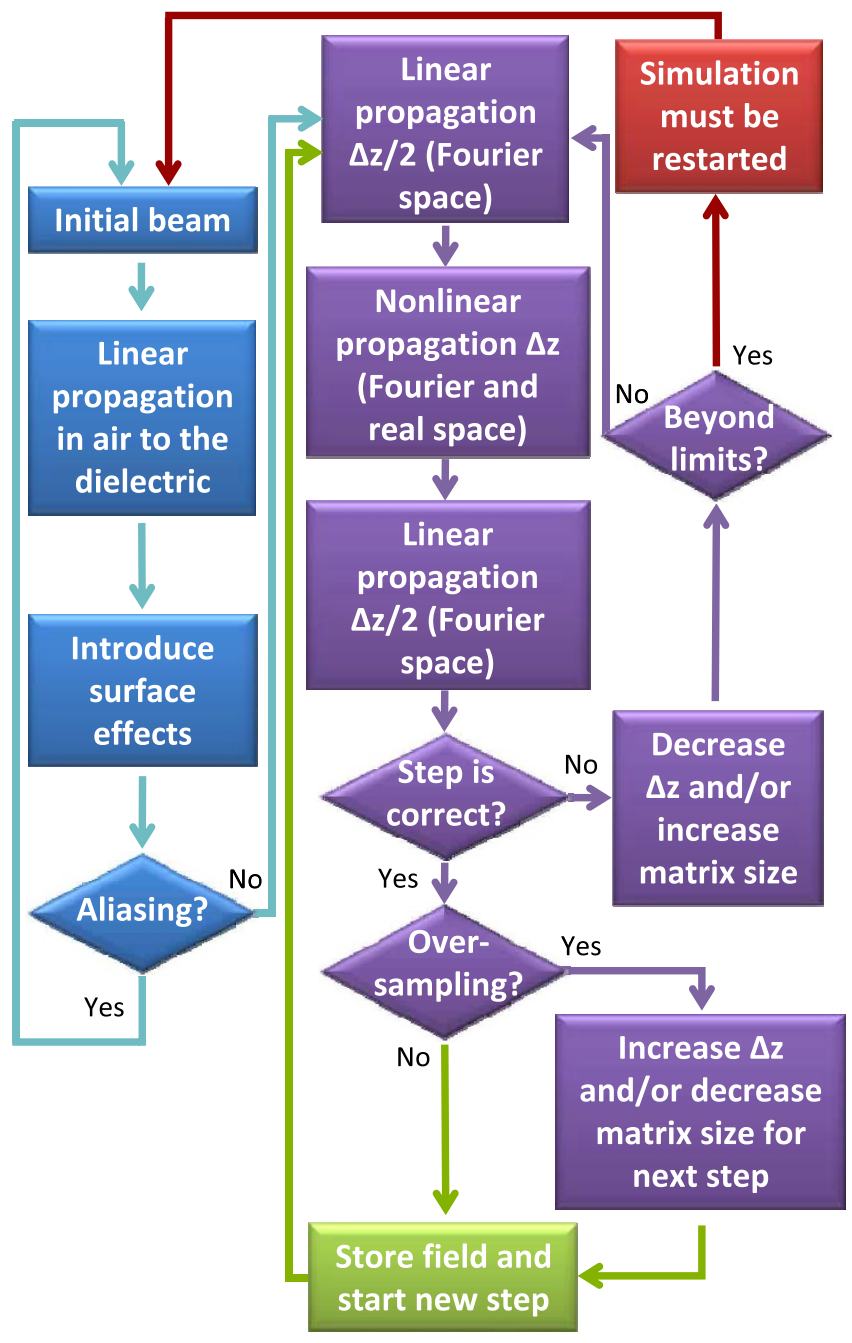

Figure 6 | Flow diagram of the beam propagation code. 
representation parameters. When the field pass both tests, the code stores the $2 \mathrm{D}$-slice $A(z+\Delta z)$ and calculates (at wish) related magnitudes as intensity, beam radius, field distribution momentums, etc. In particular, the energy absorbed in each step can be calculated and used to make an estimate of the electron density generated by multiphoton absorption, with the above indicated constrains.

Finally, when all the data is calculated from the field, the code follows to the next step until the desired depth is reached. In that moment, the code stops the calculation of the propagation and starts the representation of the desired output. It can consist of different cross sections of the beam intensity or absorbed energy, or different order momentums of the intensity, in order to access, for instance to the beam waist evolution during propagation.

1. Chiao, R. Y., Garmire, E. \& Townes, C. H. Self-trapping of optical beams. Phys. Rev. Lett. 13, 479-482 (1964).

2. Kelley, P. L. Self-focusing of optical beams. Phys. Rev. Lett. 15, 1005-1008 (1965).

3. Chiao, R. Y., Gustafson, T. K. \& Kelley, P. L. [Self-focusing: Past and Present] Topics in Appl. Phys. Vol. 114 [Boyd, R. W., Lukishova, S. G. \& Shen, Y. R. (ed.)) [129-143] (Springer New York 2009).

4. Hercher, M. Laser-induced damage in transparent media. J. Opt. Soc. Am. 54, 563-569 (1964).

5. Yablonovitvh, E. \& Bloembergen, N. Avalanche Ionization and the Limiting Diameter of Filaments Induced by Light Pulses in Transparent Media. Phys. Rev. Lett. 29, 907-910 (1972).

6. Bloembergen, N. Laser-induced electric breakdown in solids. IEEE J. Quantum Electron. 10, 375-386 (1974)

7. Couairon, A. \& Mysyrowicz, A. Femtosecond filamentation in transparent media. Phys. Rep. 441, 47-189 (2007).

8. Stegeman, G. I. \& Segev, M. Optical Spatial Solitons and Their Interactions: Universality and Diversity. Science 286, 1518-1523 (1999).

9. Couairon, A., Sudrie, L., Franco, M., Prade, B. \& Mysyrowicz, A. Filamentation and damage in fused silica induced by tightly focused femtosecond laser pulses. Phys. Rev. B 71, 125435 (2005).

10. Liu, Y. et al. Ciliary White Light: Optical Aspect of Ultrashort Laser Ablation on Transparent Dielectrics. Phys. Rev. Lett. 110, 097601 (2013).

11. Faccio, D. et al. Kerr-induced spontaneous Bessel beam formation in the regime of strong two-photon absorption. Opt.Express 16, 8213-18 (2008).

12. Locatelli, A., Pigozzo, F.-M., Modotto, D., Capobianco, A.-D. \& De Angelis, C. Bidirectional beam propagation method for multilayered dielectrics with quadratic nonlinearity". IEEE J. on Selected Topics in Quantum Electronics 8, 440-447 (2002).

13. Stegeman, G. I. \& Stegeman, R. A. Nonlinear optics: Phenomena, Materials and Devices (Wiley, (2012))

14. Sudrie, L. et al. Femtosecond laser-induced damage and filamentary propagation in fused silica. Phys. Rev. Lett. 89, 186601 (2002).

15. Kolesik, M. \& Moloney, J. Nonlinear optical pulse propagation simulation: From Maxwell's to unidirectional equations. Phys. Rev. E 70, 036604 (2004).

16. Dubietis, A., Gaižauskas, E., Tamošauskas, G. \& Di Trapani, P. Light Filaments without Self-Channeling. Phys. Rev. Lett. 92, 253903 (2004).

17. Winkler, S. W. et al. Transient response of dielectric materials exposed to ultrafast laser radiation. Appl. Phys. A 84, 413-422 (2006).

18. Pasquazi, A. et al. Near-infrared spatial solitons in heavy metal oxide glasses. Opt. Lett. 32, 2103-2105 (2007).

19. Rethfeld, B. Unified Model for the Free-Electron Avalanche in Laser-Irradiated Dielectrics. Phys. Rev. Lett. 92, 187401 (2004).

20. Christensen, B. \& Balling, P. Modeling ultrashort-pulse laser ablation of dielectric materials. Phys. Rev. B 79, 155424 (2009).

21. Ferrer, A. et al. In situ assessment and minimization of nonlinear propagation effects for femtosecond-laser waveguide writing in dielectrics. JOSA B 27, 1688-1692 (2010)

22. Femtosecond Laser Micromaching: Photonic and Microfluidic Devices in Transparent Materials [Osellame R., Cerullo G., and Ramponi R. (ed.)] Topics in Appl. Phys. Vol. 123 (Springer, Berlin, (2012)).

23. Marcinkevičius, A., Mizeikis, V., Juodkazis, S., Matsuo, S. \& Misawa, H. Effect of refractive index-mismatch on laser microfabrication in silica glass. Appl. Phys. A 76, 257-260 (2003).

24. Hnatovsky, C. et al. High-resolution study of photoinduced modification in fused silica produced by a tightly focused femtosecond laser beam in the presence of aberrations. J. Appl. Phys. 98, 013517 (2005).

25. Rayner, D., Naumov, A. \& Corkum, P. B. Ultrashort pulse non-linear optical absorption in transparent media. Opt. Express 13, 3208-17 (2005).

26. Gawelda, W. et al. Ultrafast imaging of transient electronic plasmas produced in conditions of femtosecond waveguide writing in dielectrics. Appl. Phys. Lett. 93, 121109 (2008)

27. Mishchik, K. et al. Photoinscription domains for ultrafast laser writing of refractive index changes in BK7 borosilicate crown optical glass. Opt. Mater. Express 3, 67-85 (2013).
28. Siegel, J. et al. Waveguide structures in heavy metal oxide glass written with femtosecond laser pulses above the critical self-focusing threshold. Appl. Phys. Lett. 86, 121109 (2005)

29. Grace, E. J. Asymmetric waveguide writing modeled with gaffe. OSA Technical Digest, Proceedings of "Frontiers in Optics" FThE8, 1-2 (2009).

30. Grace, E. J. GAFFE, A toolbox for solving evolutionary nonlinear PDEs (2009) Available at: www.mathworks.co.uk/matlabcentral/fileexchange/24016, (Accessed: 9th September 2014).

31. Fibich, G. \& Ilan, B. Self-focusing of elliptic beams: an example of the failure of the aberrationless approximation. J. Opt. Soc. Am. B 17, 1749 (2000).

32. Kolesik, M., Katona, G., Moloney, J. \& Wright, E. Physical Factors Limiting the Spectral Extent and Band Gap Dependence of Supercontinuum Generation. Phys. Rev. Lett. 91, 043905 (2003).

33. Schaffer, C. B., Brodeur, A. \& Mazur, E. Laser-induced breakdown and damage in bulk transparent materials induced by tightly focused femtosecond laser pulses. Meas. Sci. Technol. 12, 1784 (2001).

34. Keldysh, L. V. Ionization in the field of a strong electromagnetic wave. Sov. Phys. JETP 20, 1307 (1965).

35. Sutherland, R. L. Handbook of Nonlinear Optics (Marcel Dekker, New York, 2003).

36. Brodeur, A. \& Chin, S. L. Band-Gap Dependence of the Ultrafast White-Light Continuum. Phys. Rev. Lett. 80, 4406-4409 (1998).

37. Burakov, I. M. et al. Spatial distribution of refractive index variations induced in bulk fused silica by single ultrashort and short laser pulses. J. Appl. Phys. 101, 043506 (2007).

38. Brodeur, A. \& Chin, S. L. Ultrafast white-light continuum generation and selffocusing in transparent condensed media. JOSA B 16, 637-650 (1999).

39. Rethfeld, B. Free-electron generation in laser-irradiated dielectrics. Phys. Rev. $B$ 47, 035101 (2006).

40. Pasquazi, A., Stivala, S., Assanto, G., Gonzalo, J. \& Solis, J. Transverse nonlinear optics in heavy-metal-oxide glass. Phys. Rev. A 77, 4-9 (2008).

41. Munoz-Martin, D. et al. Structural origin of the nonlinear optical properties of lead niobium germanate film glasses. J. Appl. Phys. 110, 023522 (2011).

42. Diez-Blanco, V., Siegel, J., Ferrer, A., Ruiz de la Cruz, A. \& Solis, J. Deep subsurface waveguides with circular cross section produced by femtosecond laser writing. Appl. Phys. Lett. 91, 051104 (2007).

43. Cheng, Y. et al. Control of the cross-sectional shape of a hollow microchannel embedded in photostructurable glass by use of a femtosecond laser. Opt. Lett. 28, 55-7 (2003).

44. Blömer, D. et al. Nonlinear refractive index of fs-laser-written waveguides in fused silica. Opt. Express 14, 2151-7 (2006).

45. Temnov, V., Sokolowski-Tinten, K., Zhou, P., El-Khamhawy, a. \& von der Linde, D. Multiphoton Ionization in Dielectrics: Comparison of Circular and Linear Polarization. Phys. Rev. Lett. 97, 1-4 (2006).

46. Reiss, H. R. Polarization Effects in High-Order Multiphoton Ionization. Phys. Rev. Lett. 29, 1129-1131 (1972).

47. Ruiz de la Cruz, A. et al. Independent control of beam astigmatism and ellipticity using a SLM for fs-laser waveguide writing. Opt. Express 17, 20853-9 (2009).

\section{Acknowledgments}

This work has been partly funded by MINECO TEC2011-22422 project. J. de H. acknowledges funding from the JAE CSIC Program (pre-doctoral fellowship co-funded by the European Social Fund). A.P. acknowledges support from the People Program (Marie Curie Actions) Incoming International Fellowship (CHRONOS) under REA grant agreement $\mathrm{n}^{\circ}$ [327627].

\section{Author contributions}

J.So. and E.G. conceived the idea. J.de H., A.R., A.F. and E.G. contributed to the code development. J.de H., A.R. and A.F. performed the simulations. J.Si., A.F., A.P., G.A. and J.So. contributed to the comparison with experimental results. All authors analyzed the results, and contributed to the article. J.So. directed the project.

\section{Additional information}

Competing financial interests: The authors declare no competing financial interests.

How to cite this article: del Hoyo, J. et al. Rapid assessment of nonlinear optical propagation effects in dielectrics. Sci. Rep. 5, 7650; DOI:10.1038/srep07650 (2015)

This work is licensed under a Creative Commons Attribution-NonCommercialNoDerivs 4.0 International License. The images or other third party material in this article are included in the article's Creative Commons license, unless indicated otherwise in the credit line; if the material is not included under the Creative Commons license, users will need to obtain permission from the license holder in order to reproduce the material. To view a copy of this license, visit http:// creativecommons.org/licenses/by-nc-nd/4.0/ 